\title{
Results of a Prospective Multicenter International Atomic Energy Agency Sentinel Node Trial on the Value of SPECT/CT Over Planar Imaging in Various Malignancies
}

\author{
Amelia Jimenez-Heffernan ${ }^{1}$, Annare Ellmann², Heitor Sado $^{3}$, Dražen Huić ${ }^{4}$, Chandrasekhar Bal ${ }^{5}$,

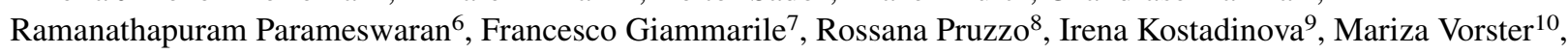 \\ Paulo Almeida ${ }^{11}$, Jonas Santiago ${ }^{12}$, Sanjay Gambhir ${ }^{13}$, Sonya Sergieva ${ }^{14}$, Alvaro Calderon ${ }^{15}$, Gabriela Oh Young ${ }^{16}$, \\ Renato Valdes-Olmos ${ }^{17}$, John Zaknun ${ }^{18}$, Vincent Peter Magboo ${ }^{19}$, and Thomas N.B. Pascual ${ }^{18}$
}

\begin{abstract}
${ }^{1}$ Hospital Juan Ramon Jimenez, Huelva, Spain; ${ }^{2}$ Stellenbosch University, Tygerberg Hospital, Stellenbosch, South Africa; ${ }^{3}$ Instituto Do Cancer de Sao Paulo, Sao Paulo, Brazil; ${ }^{4}$ University Hospital Centre Zagreb, Zagreb, Croatia; ${ }^{5}$ All India Institute of Medical Sciences, New Delhi, India; ${ }^{6}$ Manipal Hospital, Bangalore, India; ${ }^{7}$ Hospices Civils, University of Lyon 1, Lyon, France; ${ }^{8}$ Fundacion Arturo Lopez Perez, Santiago, Chile; ${ }^{9}$ University Hospital Alexandrovska, Sofia, Bulgaria; ${ }^{10}$ Pretoria Academic Hospital, Pretoria, South Africa; ${ }^{11}$ Real Hospital Português, Recife, Brazil; ${ }^{12}$ St. Lukes Medical Center, Quezon City, Philippines; ${ }^{13}$ Sanjay Gandhi Institute of Medical Sciences, Lucknow, India; ${ }^{14}$ Sofia Cancer Center, Sofia, Bulgaria; ${ }^{15}$ Instituto Nacional de Cancerlologia, Bogota, Colombia; ${ }^{16}$ Universidade de Sao Paulo, Sao Paulo, Brazil; ${ }^{17}$ The Netherlands Cancer Institute, Amsterdam, The Netherlands; ${ }^{18}$ IAEA, Vienna, Austria; and ${ }^{19}$ University of the Philippines Manila, Manila, Philippines
\end{abstract}

We aimed to assess the additional value of SPECT/CT over planar lymphoscintigraphy $(\mathrm{PI})$ in sentinel node $(\mathrm{SN})$ detection in malignancies with different lymphatic drainage such as breast cancer, melanoma, and pelvic tumors. Methods: From 2010 to 2013, 1,508 patients were recruited in a multicenter study: 1,182 breast cancer, 262 melanoma, and 64 pelvic malignancies (prostate, cervix, penis, vulva). PI was followed by SPECT/CT 1-3 h after injection of 99mTccolloid particles. Surgery was performed the same or next day. Results: Significantly more SNs were detected by SPECT/CT for breast cancer $(2,165$ vs. 1,892$)$, melanoma (602 vs. 532 ), and pelvic cancer (195 vs. 138), all $P<0.001$. The drainage basin mismatch between PI and SPECT/CT was $16.5 \%$ for breast cancer, $11.1 \%$ for melanoma, and $51.6 \%$ for pelvic cancers. Surgical adjustment was $17 \%$ for breast cancer, $37 \%$ for melanoma, and $65.6 \%$ for pelvic cancer. Conclusion: SPECT/CT detected more SNs and changed the drainage territory, leading to surgical adjustments in a considerable number of patients in all malignancies studied but especially in the pelvic cancer group because of this group's deep lymphatic drainage. We recommend SPECT/CT in all breast cancer patients with no SN visualized on $\mathrm{PI}$, all patients with melanoma of the head and neck or trunk, all patients with pelvic malignancies, and those breast cancer and melanoma patients with unexpected drainage on $\mathrm{PI}$.

Key Words: sentinel node; SPECT/CT; breast cancer; melanoma; pelvic cancer

J Nucl Med 2015; 56:1338-1344

DOI: 10.2967/jnumed.114.153643
$\mathbf{T}$ he status of regional lymph nodes is a major prognostic factor in many malignant tumors. Sentinel lymph node (SN) biopsy in patients with clinically node-negative tumors is a validated technique for accurate staging of nodal disease in breast cancer $(1,2)$ and melanoma $(3,4)$ and is being used with promising results in other solid tumors including pelvic malignancies $(5,6)$. The use of SN mapping in an increasing list of tumors shows a variety of lymphatic drainage basins containing the SN (7). SNs are the lymph nodes draining the primary tumor and therefore the most likely to contain tumor cells spreading to the drainage basin (8). Assuming an orderly progression of lymph flow, the tumor status of the SN predicts the status of the regional draining basin (1). Selective $\mathrm{SN}$ biopsy enables the detection of metastatic and occult micrometastatic nodal involvement by thorough histopathologic examination in the intraoperative setting, sparing patients a systematic regional lymphadenectomy when the node is negative.

Planar lymphoscintigraphy (PI) after local injection of radiocolloid is well suited for the mapping of regional lymph nodes, but occasionally no SNs are visualized, for instance, in obese patients or when SNs are located too close to the injection site. In addition, the anatomic information provided by planar scintigraphy is limited and the exact SN location is difficult to define in deeply located nodes $(9,10)$. Unexpected basin drainage sites and unpredictable pathways also hinder interpretation of planar images. Because of its superior contrast, resolution, and display of exact anatomic landmarks, SPECT/CT can overcome most of these limitations (11-18).

In the present study we aimed to assess the additional value of SPECT/CT over PI in a large series of patients with tumors draining to different lymphatic drainage basins throughout the body.

\section{MATERIALS AND METHODS}

From December 2010 to September 2013, 1,508 patients were recruited in a prospective multicenter research project of the International Atomic Energy Agency (IAEA) conducted at 15 centers from 10 different countries. Each institutional review board approved this study, and all 
subjects signed a written informed consent form. Tables 1 and 2 display patient characteristics and patients per site. Inclusion criteria were invasive/in situ breast cancer (T1-4), melanoma with a Breslow thickness of 0.75-4 $\mathrm{mm}$ and pelvic carcinoma with a tumor size of $3 \mathrm{~cm}$ or smaller, and all N0 and M0. Exclusion criteria were pregnancy, inflammatory breast cancer, palpable node, age below $18 \mathrm{y}$, and refusal to give consent.

\section{General Protocol}

Dual-head SPECT/CT $\gamma$ cameras with low-energy high-resolution collimators were used (Infinia-Hawkeye [GE Healthcare], Symbia T2T16 [Siemens], and Precedence 6 [Philips]). PI was performed with 5 -min static images obtained in anterior, posterior, oblique, and lateral views as deemed adequate (matrix, $256 \times 256$; prostate cancer, $512 \times$ 512). A ${ }^{57} \mathrm{Co}$ flood source was placed to delineate the body contour. SPECT/CT was performed immediately after planar acquisition, comprising between 80 and 120 views of $20-40$ s (matrix, $128 \times 128$; cervical cancer, $64 \times 64)$. For SPECT/CT, the patient was supine with the arms above the head in breast cancer and along the body sides in the rest. Reconstruction was iterative using ordered-subset expectation maximization algorithms. CT data were used for attenuation correction and anatomic localization of lymph nodes. First draining nodes in a basin and all lymph nodes visualized with a lymphatic duct draining from the injection site were considered SNs. Lymph nodes depicted in basins different from those of the first draining nodes were also considered SNs. The number of SNs and their draining basin localization were evaluated separately on planar and SPECT/CT images to establish the incremental value of SPECT/CT over planar scintigraphy (additional SNs or mismatch in localization). In patients with superficial drainage, the site of SNs was marked on the skin with a felt-tip pen. Planar and SPECT/CT images were discussed with the surgeon.

Lymphoscintigraphy was performed after injection of ${ }^{99 \mathrm{~m}}$ Tc-labeled colloid particles, Nanocoll (GE Healthcare) in most cases (breast carcinoma, 39.8\%; melanoma, 36.5\%; pelvis, $100 \%$ ). The injection site was gently massaged for a few minutes immediately after injection in breast cancer and melanoma patients, and movement of the ipsilateral arm was encouraged before imaging in breast cancer patients. Table 3 displays the injected doses and volumes.

\section{Breast Cancer}

Breast cancer was present in 1,182 patients. Axillary ultrasonography was performed in patients with clinically negative axillae, and suspected nodes underwent fine-needle aspiration cytology. Radiopharmaceutical injection was periareolar, peritumoral, or both in $58.2 \%$, $28.2 \%$, and $13.6 \%$ of patients, respectively. Injection was guided by ultrasonography in $26.2 \%$ of cases.

\section{TABLE 1}

Patient Characteristics

\begin{tabular}{lccc}
\hline \multicolumn{1}{c}{ Characteristic } & Breast & Melanoma & Pelvis \\
\hline No. of patients & 1,182 & 262 & $\begin{array}{c}\text { Prostate, 40 } \\
\text { Cervix, 15 } \\
\end{array}$ \\
& & & $\begin{array}{c}\text { Penis, } 7 \\
\text { Vulva, } 2\end{array}$ \\
\hline Mean age (y) & $55.2 \pm 12.3$ & $53.9 \pm 15.2$ & $60 \pm 11.3$ \\
\hline Sex & & & \\
\hline Male & 7 & 117 & 47 \\
\hline Female & 1,175 & 145 & 17 \\
\hline Body mass index & $25.1 \pm 8.2$ & $24.6 \pm 8.7$ & $27 \pm 3.9$ \\
\hline
\end{tabular}

TABLE 2

Patients per Site

\begin{tabular}{|lccc}
\hline \multicolumn{1}{c}{ Site } & Breast & Melanoma & Pelvis \\
\hline Brazil (1) & 230 & 40 & \\
\hline Spain & 268 & & \\
India (1) & 135 & & \\
Croatia & 79 & 38 & 1 \\
\hline India (2) & 109 & & \\
\hline Chile & 53 & 42 & \\
Bulgaria (1) & 48 & 44 & \\
\hline South Africa (1) & 38 & 38 & \\
\hline Brazil (2) & 36 & 40 & \\
\hline Philippines & 72 & & \\
\hline India (3) & 59 & & \\
\hline Bulgaria (2) & 46 & 9 & \\
\hline The Netherlands & & & \\
\hline Colombia & 9 & 11 & \\
\hline South Africa (2) & & & \\
\hline
\end{tabular}

Data in parentheses are sites per country.

\section{Melanoma}

Melanoma of intermediate thickness (Breslow, 0.75-4 mm) and no evidence of palpable lymph nodes were present in 262 patients. The radiopharmaceutical was administered intradermally within $1 \mathrm{~cm}$ from the tumor or biopsy site. A 20- to 30-min dynamic study comprising 0.5- to 1-min frames was acquired immediately after injection. For head and neck melanomas, the dynamic acquisition comprised 15-s frames for 15 min. Subsequently, planar and SPECT/CT images were acquired. Surgery was the same day in $47.2 \%$ of patients and the day after in $52.8 \%$.

\section{Pelvic Cancer}

Pelvic cancer with no evidence of abnormal enlarged lymph nodes was present in 64 patients. In prostate cancer patients, intraprostatic injections ( 2 into each lobe) were given under the guidance of transrectal ultrasound followed by flushing with $0.7 \mathrm{~mL}$ of saline. Patients received antibiotic therapy to prevent prostatitis.

Planar images were acquired $15 \mathrm{~min}$ and $2 \mathrm{~h}$ after injection and SPECT/CT after the late planar views. Surgery was performed the same day in all patients. Cervical cancer patients received superficial peritumoral (intradermal/submucosal) injections of the radiopharmaceutical. In patients with penile or vulvar cancer, ${ }^{99 \mathrm{~m}} \mathrm{Tc}$-labeled colloid particles were injected after preparation with anesthetic spray. A 45-min dynamic study comprising 1-min frames was performed immediately after injection. Next, PI and SPECT/CT were performed. Surgery was the same day in $83.3 \%$ of the patients and the day after in $16.7 \%$.

\section{Statistical Methods}

Results are presented as the mean \pm SD for continuous variables and frequencies (proportions) for categoric variables. Comparisons were performed using paired $t$ test. A 2-tailed $P$ value of less than 0.05 was considered significant for all tests. Calculations were performed using SPSS Statistics (version 20; IBM).

\section{RESULTS}

Significantly more SNs were detected by SPECT/CT than by PI in the 3 groups of patients $(P<0.001)$ (Table 4$)$. There were no 
TABLE 3

Dose and Injection Details

\begin{tabular}{lcrc}
\hline Patient & Breast & Melanoma & Pelvis \\
\hline Injected dose $(\mathrm{MBq})$ & $61.4 \pm 28.2$ & $50.4 \pm 27.4$ & Prostate, $214.2 \pm 11.6$ \\
& & & Cervix, $67.6 \pm 18.8$ \\
& & & Penis/vulva, $40.2 \pm 9.8$ \\
\hline Injected volume $(\mathrm{mL})$ & $0.38 \pm 0.34$ & $0.49 \pm 0.31$ & Prostate, $0.4 \pm 0.0$ \\
& & & Cervix, $0.4 \pm 0.3$ \\
\hline No. of injection sites & & & Penis/vulva, $0.5 \pm 0.2$ \\
1 & $824(69.7)$ & $7(2.7)$ & - \\
2 & $212(17.9)$ & $16(6.1)$ & - \\
3 & $53(4.5)$ & $13(5.0)$ & $7(10.9)$ \\
4 & $80(6.8)$ & $223(85.1)$ & $57(89.1)$ \\
6 & $13(1.1)$ & $1(0.4)$ & - \\
\end{tabular}

Data are mean \pm SD or $n$ (no. of patients), with percentages in parentheses.

significant differences in age and body mass index between patients with and without SN detection on either PI or SPECT/CT. Patients' age and body mass index did not differ significantly among the different tumor groups. Tables 4 and 5 summarize results on an SN and patient basis, respectively.

\section{Breast Cancer}

Planar images failed to visualize SNs in 146 patients (12.3\%). Of these, SPECT/CT depicted 1 or more SNs (Fig. 1) in $44(3.7 \%)$ patients, with persistent nonvisualization in 102 patients $(8.6 \%)$. Of these, 56 patients $(54.9 \%)$ had metastatic nodes on the axillary lymphadenectomy. The location of the 273 additional SNs found in 232 patients was axillary in 134 patients, interpectoral in 34, internal mammary in 27 , intramammary in 20 , clavicular in 15 , contralateral axillary in 1 , and contralateral internal mammary in 1 . SPECT/CT showed additional SNs in 112 of the 195 patients with drainage mismatch, the remaining 83 patients showing the same number of SNs on either modality. In the latter, the most frequent adjustments in drainage territory from PI to SPECT/CT were from axillary to interpectoral (44.6\%) and intramammary $(21.7 \%)$ locations.

The locations of the positive-for-metastases SNs depicted only by SPECT/CT in 38 patients were axillary, intramammary, interpectoral, and infraclavicular in 27, 4, 4, and 3 patients, respectively. In 33 of these patients, the SN depicted only on SPECT/CT was the only positive node. Internal mammary and other extraaxillary SNs (Fig. 2) were not routinely excised in all centers.

\section{Melanoma}

No SN was visualized on PI in 3 patients. SPECT/CT revealed an axillary $\mathrm{SN}$ in a trunk melanoma patient and persistent nonvisualization in 2 patients with head and neck melanoma. In the latter, cervical lymphadenectomy was positive for metastases. The locations of the 70 additional SNs revealed by SPECT/CT in 53 patients were levels IB, IIB, III, intraparotid, and occipital in 6 patients with head and neck melanoma (25\%); axillary and clavicular in 11 patients with upper limb melanoma (25.5\%); axillary, clavicular, and scapular in 29 patients with trunk melanoma (20.5\%); and inguinal and popliteal in 7 patients with lower limb melanoma (12.9\%). SPECT/CT showed additional SNs in 13 of the 29 patients with drainage mismatch. The 2 patients with a positive SN depicted only by SPECT/CT had trunk melanomas. The locations were axillary and inguinal, the latter being the only positive node in the patient. The surgical approach was modified in 97 patients (37\%): $41.6 \%, 39.7 \%, 33.3 \%$, and $30.2 \%$ of head and neck, trunk, lower limb, and upper limb lesions, respectively.

\section{Pelvic Cancer}

In the prostate cancer group, no SNs were depicted on PI in 4 patients whereas SPECT/CT revealed 1 or more SNs. Locations

TABLE 4

PI and SPECT/CT SN Findings

\begin{tabular}{|c|c|c|c|}
\hline SN detection & Breast cancer & Melanoma & Pelvic cancer \\
\hline Planar & 1,892 & 532 & 138 \\
\hline SPECT/CT & 2,165 & 602 & 195 \\
\hline Planar and SPECT/CT & 1,871 & 522 & 138 \\
\hline Mean planar SN & $1.6 \pm 1.3$ & $2 \pm 1.3$ & $2.1 \pm 1.3$ \\
\hline Mean SPECT/CT SN & $1.8 \pm 1.4$ & $2.3 \pm 1.4$ & $3 \pm 1.65$ \\
\hline Positive SNs with SPECT/CT but not planar & 44 & 3 & 4 \\
\hline
\end{tabular}


TABLE 5

Patients' SN Details and Surgical Impact

\begin{tabular}{|c|c|c|c|}
\hline No. of patients (\%) with... & $\begin{array}{l}\text { Breast cancer } \\
\quad(n=1,182)\end{array}$ & $\begin{array}{c}\text { Melanoma } \\
(n=262)\end{array}$ & $\begin{array}{l}\text { Pelvic cancer } \\
\quad(n=64)\end{array}$ \\
\hline SN found & $1,080(91.4)$ & $260(99.2)$ & $61(95.3)$ \\
\hline No SN found & $102(8.6)$ & $2(0.8)$ & $3(4.7)$ \\
\hline Planar detection rate* & $1,036(87.6)$ & $259(98.8)$ & $56(87.5)$ \\
\hline SPECT/CT detection rate* & $1,080(91.4)$ & 260 (99.2) & $61(95.3)$ \\
\hline At least $1 \mathrm{SN}$ found with SPECT/CT but none with planar & $44(3.7)$ & $1(0.4)$ & $5(7.8)$ \\
\hline Equal SNs with planar and SPECT/CT & $929(78.6)$ & $199(75.9)$ & $29(45.3)$ \\
\hline More SNs with planar than SPECT/CT ${ }^{\dagger}$ & $21(1.8)$ & $10(3.8)$ & 0 \\
\hline \multirow[t]{5}{*}{ More SNs with SPECT/CT than planar } & $232(19.6)$ & $53(20.2)$ & All, 35 (54.6) \\
\hline & & & Prostate, 33 (82.5) \\
\hline & & & Cervix, 1 (6.6) \\
\hline & & & Penis, 1 (14.2) \\
\hline & & & Vulva, 0 \\
\hline No drainage basin mismatch & $987(83.5)$ & $233(88.9)$ & $31(48.4)$ \\
\hline Drainage basin mismatch & $195(16.5)$ & $29(11.1)$ & $33(51.6)$ \\
\hline At least $1 \mathrm{SN}$ positive for metastasis & $366(31)$ & $34(13)$ & $16(25)$ \\
\hline At least $1 \mathrm{SN}$ positive for metastasis planar & $328(29.7)$ & $34(13)$ & $13(20.3)$ \\
\hline At least $1 \mathrm{SN}$ positive for metastasis SPECT/CT & $366(31)$ & $33(12.6)$ & $16(25)$ \\
\hline At least $1 \mathrm{SN}$ positive for metastasis planar but not SPECT/CT & 0 & 0 & 0 \\
\hline At least $1 \mathrm{SN}$ positive for metastasis SPECT/CT but not planar & $38(3.2)$ & $2(0.8)$ & $2(3.1)$ \\
\hline Surgical adjustment $\ddagger$ & $200(17)$ & $97(37)$ & $42(65.6)$ \\
\hline Without changes in management if only SPECT/CT performed & 0 & 0 & 0 \\
\hline \multicolumn{4}{|c|}{$\begin{array}{l}{ }^{\star} \text { At least } 1 \mathrm{SN} \text { found. } \\
\text { †Planar SN finding revealed by SPECT/CT to be skin contamination or hold up in lymphatic channels. } \\
\text { FPatients without changes in management if only planar imaging had been performed. }\end{array}$} \\
\hline
\end{tabular}

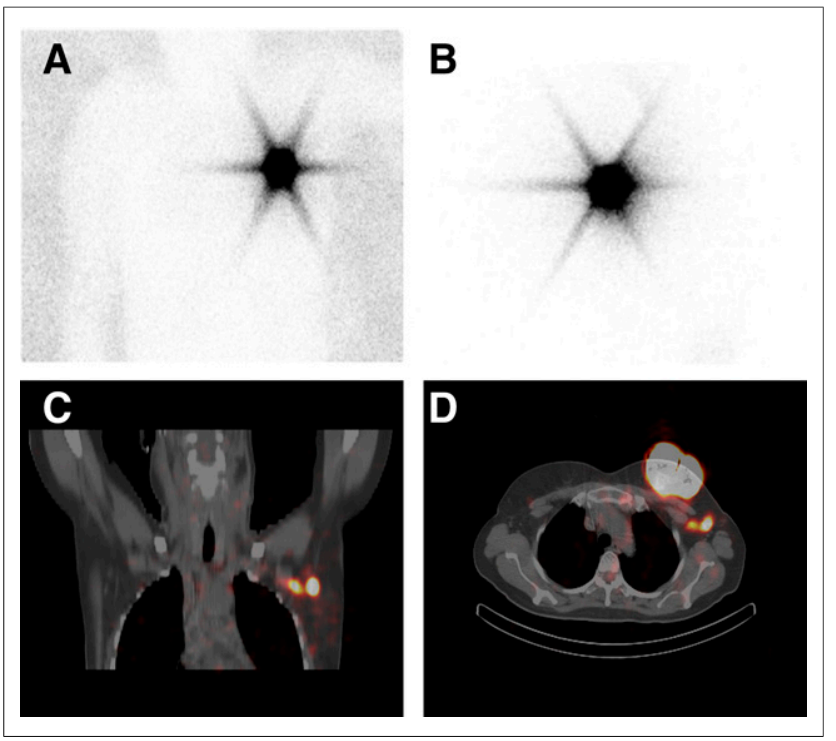

FIGURE 1. Patient with left breast cancer in upper inner quadrant of small breast. No lymphatic drainage is seen on anterior (A) and lateral (B) planar images. SPECT/CT reveals 2 SNs right behind tumor (C, coronal view; D, transaxial view). were obturator in 1 patient and internal iliac and obturator in the other 3 . The locations of the 57 additional nodes revealed in 33 patients, with mismatch versus PI in 32 , were obturator (17), common iliac (13), pararectal (6), presacral (6), internal iliac (4), paravesical (2), paraaortic (2), psoas muscle (2), near the abdominal wall (2), external iliac (2), and aortocaval (1) (Fig. 3). In the 7 patients with the same number of SNs on planar and SPECT/CT imaging, the latter provided a more precise localization of the $\mathrm{SN}$ in the obturator fossa, common iliac, and external iliac basin. SPECT/CT information modified the surgical approach in 33 patients $(82.5 \%)$.

In the cervical cancer group, PI did not show SNs in 4 patients, for whom SPECT/CT showed an SN in 1 patient and persistent nonvisualization in the other 3 . The localization of this additional SN was left mesorectal at the level of the sacrococcygeal joint. In the remaining 11 patients, there were no additional SNs visualized on SPECT/CT. Although SPECT/CT information changed only the surgical approach in the case with the additional SN on SPECT/CT, it increased surgeon confidence in all other cases. PI and SPECT/CT showed SNs in all cases in the penile cancer group. SPECT/CT depicted an extra right inguinal SN in a patient with 2 inguinal nodes (bilateral) seen on PI. The surgical incision was modified in this and in 5 additional patients (total, 85\%) without extra SNs due to better localization of the SNs, leading to a more precise incision. In the vulva patients, inguinal SNs 

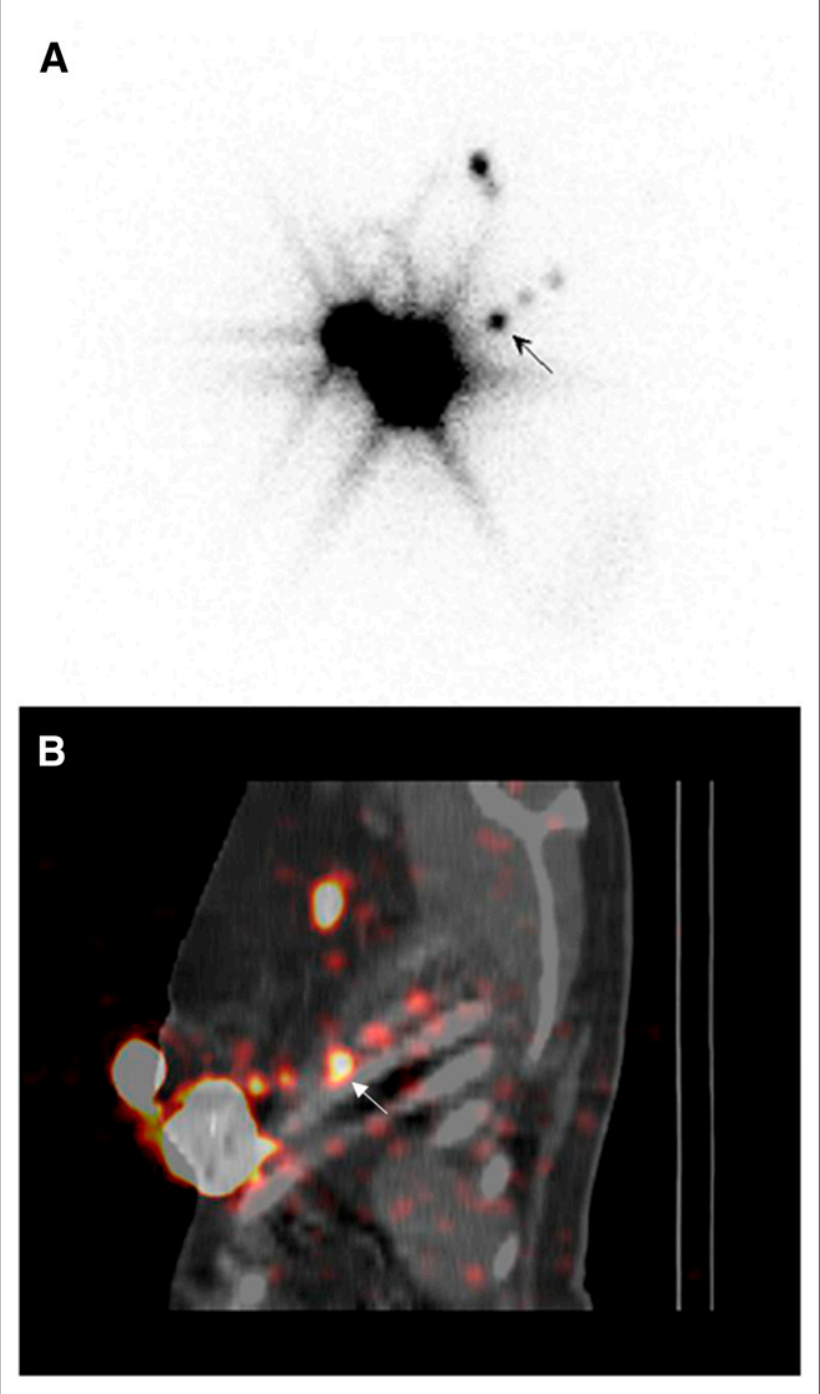

FIGURE 2. Patient with left breast cancer in lower outer quadrant. (A) Lateral planar view reveals axillary drainage and lateral nodes of uncertain location (arrow). SPECT/CT clearly depicts 3 consecutive costal nodes (arrow) in addition to the axillary nodes. Sagittal view is depicted in $\mathrm{B}$.

were seen on PI with no extra SNs visualized with SPECT/CT; nevertheless, the surgical incision was changed due to better localization.

\section{DISCUSSION}

Planar lymphoscintigraphy is a robust technique able to provide a roadmap for surgeons by tracing the direct lymphatic pathway from the tumor site to the SN. Its validation in malignancies with simple and superficial lymphatic drainage patterns such as melanoma, breast cancer, penile cancer, and vulvar cancer has been easier than for gastrointestinal, urological, and gynecologic malignancies with deeply located SNs (12). For melanomas of head and neck or trunk, SN localization in the operating room may become difficult because of a more complex anatomy. Also, extraaxillary SNs in breast cancer represent a surgical challenge and cannot always be resected (13). Obesity and $\mathrm{SN}$ location near the tumor injection site are additional factors that hamper detection by planar images (16). In the past decennium hybrid cameras enabling SPECT/CT were introduced and are now widely available, enabling fusion images that clearly depict the SNs in an anatomic relationship with the neighboring vascular structures and surrounding tissues. SPECT/CT, however, has a higher cost, increases camera time with related patient discomfort and limited workflow, and delivers a small but nonnegligible radiation dose $(16,19)$. In addition, the benefit of retrieving additional SNs has been questioned because more than 3 SNs increases surgery time and pathology costs without significant improvement in diagnostic accuracy in breast cancer patients $(14,20)$.

In our multicenter study, PI identified SNs in $87.6 \%, 98.8 \%$, and $87.5 \%$ of breast cancer, melanoma, and pelvic cancer patients, respectively. These visualization rates are in accordance with the reported $63 \%-88 \%$ for breast cancer $(14,16,17), 82 \%-99 \%$ for melanoma (18), and $84 \%-91 \%$ for gynecologic and prostate cancers $(6,7,21)$. SPECT/CT improved the visualization rates to $91.3 \%$ for breast cancer, $99.2 \%$ for melanoma, and $95.3 \%$ for pelvic cancer, again in agreement with the reported rates of $81 \%-97.3 \%$ for breast cancer (16-18), near $100 \%$ for melanoma (18), and $92.2 \%-100 \%$ for gynecologic and prostate cancers $(6,7,21,22)$. SPECT/CT depicted SNs in $3.7 \%$ of breast cancer, $0.4 \%$ of melanoma, and $4.7 \%$ of pelvic cancer patients, respectively, with no SNs visualized on PI, representing a considerable number of patients who would have undergone lymphadenectomy if only PI was performed. In addition, the metastatic involvement of SNs depicted only by SPECT/CT in $3.2 \%$ of breast cancer, $0.8 \%$ of melanoma, and $3.1 \%$ of pelvic cancer patients is also relevant because these patients would have been inadequately staged if SPECT/CT was not performed.

In breast cancer patients, it is important to note that SPECT/CT indicated the location of the interpectoral, intramammary, internal mammary, and other extraaxillary SNs. Interpectoral lymph nodes are in many cases the primary drainage station in breast cancer, representing a pathway by which skip metastasis may occur, and

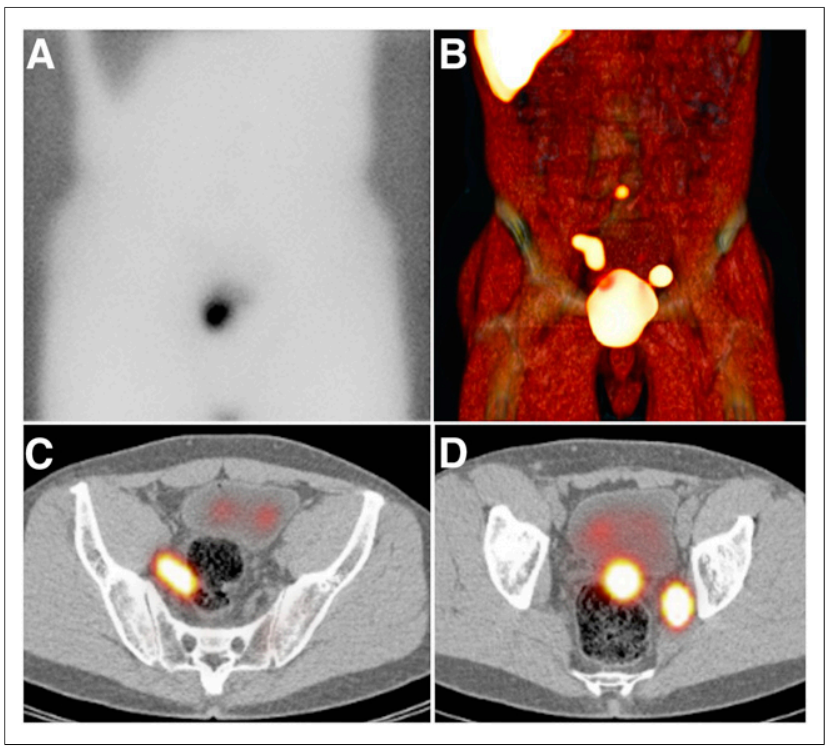

FIGURE 3. (A) Patient with prostate cancer without evident lymphatic drainage on anterior planar image. SPECT/CT displayed with volume rendering (B) shows bilateral drainage to obturator lymph nodes in pelvis and to subaortic lymph node. lliac SNs are seen as hot spots on transversal SPECT/CT fusion images (C and D). 
a potential site of recurrence. The identification of intramammary nodes has clinical implications because these nodes may contain metastases in approximately $10 \%$ of operable breast cancers and have been associated with poor disease-free survival and outcome. The internal mammary chain is the second most frequent drainage basin from breast cancer after the axilla (23), but excision of these nodes is difficult and many surgical teams do not resect them. Nevertheless, awareness of such drainage is important because it can alter the patient's management (inclusion in the area of irradiation, systemic therapy) (24). However, because of the particular drainage of the breast, the detection of internal mammary nodes has clinical relevance only if dose injection is near the primary tumor (25).

Melanomas of the limbs have predictable drainage patterns to the axilla or groin. In a limited number of cases they drain to popliteal or epitrochlear lymph nodes. However, head and neck melanomas and melanomas located near the midline and in the posterior trunk have variable and unpredictable drainage patterns with pathways not always leading to the nearest basin or leading to multiple basins $(18,26)$. In 5 of our cases, the identification of additional scapular and occipital SNs was possible only with SPECT/CT, a notable fact as they must be considered as true SNs (27).

Lymphatic drainage from the prostate is generally to the pelvic nodes, but direct drainage outside the pelvic area is a frequent finding (6). Paraaortic nodes are difficult to locate with precision by PI (7) and abdominal wall nodes may not be seen at all (26). In our study, SPECT/CT identified drainage outside the pelvic area (presacral, aortocaval, paraaortic, abdominal wall, and psoas muscle) not visualized by PI in $22.5 \%$ of patients; the aortocaval node was 1 of the metastatic SNs depicted only by SPECT/CT. Furthermore, SPECT/CT showed SNs outside the obturator fossa in $82.5 \%$ with drainage exclusively outside the obturator area in $22.5 \%$, information relevant to the extension of pelvic lymphadenectomy. Also, the depiction of paravesical and presacral SNs, exclusively identified by SPECT/CT, is important because these basins are not included in the area of extended pelvic lymphadenectomy, which is frequently considered as the modality of choice to stage the pelvis $(28)$.

The information provided by SPECT/CT modified the surgical approach in $17 \%$ of breast cancer, $37 \%$ of melanoma, and $65.6 \%$ of pelvic cancers. The lower-than-reported (10) adjustment rate in breast cancer patients may be explained by the fact that internal mammary and other extraaxillary SNs were not excised routinely in all centers. The global melanoma adjustment rate agrees with previous study results $(19,26,29)$, together with the finding of the greatest benefit for patients with head and neck (30) and trunk (26) locations. This is not surprising because prediction of the lymphatic drainage pathways of melanoma of the head and neck is difficult in a region with more than 350 small lymph nodes and fast tracer uptake (31). SPECT/CT excluded nonnodal false-positive sites of uptake in 21 breast cancer and 10 melanoma patients, avoiding unnecessary surgical search. Although the global surgical adjustment rate of pelvic cancer patients was $65.6 \%$, it was actually higher for prostate $(82.5 \%)$ and for penile and vulvar cancer $(88.8 \%)$ patients.

The advantage of this study is that it has been performed prospectively by different institutions around the world, using different techniques. Despite this, the results are similar to previously published results, confirming the accuracy of the research. A limitation of the study is the small number of patients with pelvic malignancies; there were no patients with testicular or endometrial cancer and only 2 patients with vulvar cancer.

\section{CONCLUSION}

SPECT/CT is of great benefit in patients showing no lymphatic drainage on PI, in patients with malignancies located in areas expected to show unpredictable drainage, and in cases expected to drain to deep intraabdominal or retroperitoneal drainage. On the basis of the results of this study, we recommend that SPECT/CT be performed in all patients with breast cancer and no planar SN visualization, all patients with melanoma of head and neck or trunk, and all pelvic cancer patients as well as in those breast cancer and melanoma patients with unexpected drainage on PI.

\section{DISCLOSURE}

The costs of publication of this article were defrayed in part by the payment of page charges. Therefore, and solely to indicate this fact, this article is hereby marked "advertisement" in accordance with 18 USC section 1734. This Coordinated Research Project was supported by the International Atomic Energy Agency (IAEA) under the Coordinated Research Activities EI3037 The Use of Sentinel Lymph Node in Breast, Melanoma, Head \& Neck and Pelvic Cancers approved September 2, 2010. No other potential conflict of interest relevant to this article was reported.

\section{REFERENCES}

1. Mariani G, Moresco L, Viale G, et al. Radioguided sentinel lymph node biopsy in breast cancer surgery. J Nucl Med. 2001;42:1198-1215.

2. Veronesi U, Paganelli G, Viale G, et al. A randomized comparison of sentinelnode biopsy with routine axillary dissection in breast cancer. $N$ Engl J Med. 2003;349:546-553.

3. Uren RF, Howman-Giles RB, Shaw HM, Thompson JF, Mccarthy WH. Lymphoscintigraphy in high-risk melanoma of the trunk: predicting draining node groups, defining lymphatic channels and locating the sentinel node. J Nucl Med. 1993;34:1435-1440.

4. Mariani G, Gipponi M, Moresco L, et al. Radioguided sentinel lymph node biopsy in malignant cutaneous melanoma. J Nucl Med. 2002;43:811-827.

5. Belhocine TZ, Prefontaine M, Lanvin D, et al. Added-value of SPECT/CT to lymphatic mapping and sentinel lymphadenectomy in gynaecological cancers. Am J Nucl Med Mol Imaging. 2013;3:182-193.

6. Vermeeren L, Valdés Olmos RA, Meinhardt W, et al. Value of SPECT/CT for detection and anatomic localization of sentinel lymph nodes before laparoscopic sentinel node lymphadenectomy in prostate carcinoma. J Nucl Med. 2009; 50:865-870.

7. Vermeeren L, Meinhardt W, Bex A, et al. Paraaortic sentinel lymph nodes: toward optimal detection and intraoperative localization using SPECT/CT and intraoperative real-time imaging. J Nucl Med. 2010;51:376-382.

8. Giuliano AE, Kirgan DM, Guenther JM, Morton DL. Lymphatic mapping and sentinel lymphadenectomy for breast cancer. Ann Surg. 1994;220:391398.

9. van der Ploeg IMC, Valdes Olmos RA, Kroon BBR, Rutgers EJT, Nieweg OE. The hidden sentinel node and SPECT/CT in breast cancer patients. Eur J Nucl Med Mol Imaging. 2009;36:6-11.

10. van der Ploeg IMC, Nieweg OE, Kroon BBR, et al. The yield of SPECT/CT for anatomical lymphatic mapping in patients with breast cancer. Eur J Nucl Med Mol Imaging. 2009;36:903-909.

11. van der Ploeg IMC, Valdés Olmos RA, Kroon BBR, Nieweg OE. The hybrid SPECT/CT as an additional lymphatic mapping tool in patients with breast cancer. World J Surg. 2008;32:1930-1934.

12. Zaknun JJ, Giammarile F, Olmos RV, Vidal-Sicart S, Mariani G. Changing paradigms in radioguided surgery and intraoperative imaging: the GOSTT concept. Eur J Nucl Med Mol Imaging. 2012;39:1-3.

13. Olmos RA, Vidal-Sicart S, Nieweg OE. SPECT-CT and real-time intraoperative imaging: new tools for sentinel node localization and radioguided surgery? Eur $J$ Nucl Med Mol Imaging. 2009;36:1-5.

14. Vermeeren L, Van der Ploeg IMC, Valdes Olmos RA, et al. SPECT/CT for preoperative sentinel node localization. J Surg Oncol. 2010;101:184-190. 
15. Vidal-Sicart S, Brouwer OR, Valdés-Olmos RA. Evaluation of the sentinel lymph node combining SPECT/CT with the planar image and its importance for the surgical act. Rev Esp Med Nucl. 2011;30:331-337.

16. Kraft O, Havel M. Sentinel lymph nodes and planar scintigraphy and SPECT/CT in various types of tumours: estimation of some factors influencing detection success. Nucl Med Rev Cent East Eur. 2013;16:17-25.

17. Vercellino L, Ohnona J, Groheux D, et al. Role of SPECT/CT in sentinel lymph node detection in patients with breast cancer. Clin Nucl Med. 2014;39:431-436.

18. Wagner T, Buscombe J, Gnanasegaran G, Navalkissoor S. SPECT/CT in sentinel node imaging. Nucl Med Commun. 2013;34:191-202.

19. Fairbairn N, Munson C, Khan ZA, Butterworth M. The role of hybrid SPECT/CT for lymphatic mapping in patients with melanoma. J Plast Reconstr Aesthet Surg. 2013;66:1248-1255.

20. Lynch MA, Jackson J, Kim JA, Leeming RA. Optimal number of radioactive sentinel lymph nodes to remove for accurate axillary staging of breast cancer. Surgery. 2008;144:525-531.

21. Klapdor R, Mücke J, Schneider M, et al. Value and advantages of preoperative sentinel lymph node imaging with SPECT/CT in cervical cancer. Int J Gynecol Cancer. 2014;24:295-302.

22. de Bonilla-Damiá A, Brouwer OR, Meinhardt W, Valdés-Olmos RA. Lymphatic drainage in prostate carcinoma assessed by lymphoscintigraphy and SPECT/CT: its importance for the sentinel node procedure. Rev Esp Med Nucl Imagen Mol. 2012;31:66-70.

23. Lerman H, Metser U, Lievshitz G, Sperber F, Shneebaum S, Even-Sapir E. Lymphoscintigraphic sentinel node identification in patients with breast cancer: the role of SPECT-CT. Eur J Nucl Med Mol Imaging. 2006;33:329-337.
24. Chen RC, Lin NU, Golshan M, Harris JR, Bellon JR. Internal mammary nodes in breast cancer: diagnosis and implications for patient management-a systematic review. J Clin Oncol. 2008;26:4981-4989.

25. Giammarile F, Alazraki N, Aarsvold JN, et al. The EANM and SNMMI practice guideline for lymphoscintigraphy and sentinel node localization in breast cancer. Eur J Nucl Med Mol Imaging. 2013;40:1932-1947.

26. van der Ploeg IMC, Valdés Olmos RA, Kroon BBR, et al. The yield of SPECT/ CT for anatomical lymphatic mapping in patients with melanoma. Ann Surg Oncol. 2009;16:1537-1542.

27. Alvarez Paez AM, Brouwer OR, Veenstra HJ, et al. Decisive role of SPECT/CT in localization of unusual periscapular sentinel nodes in patients with posterior trunk melanoma: three illustrative cases and a review of the literature. Melanoma Res. 2012;22:278-283.

28. Vermeeren L, Meinhardt W, Valdes Olmos RA. Prostatic lymphatic drainage with sentinel nodes at the ventral abdominal wall visualized with SPECT/CT. A case series. Clin Nucl Med. 2010;35:71-73.

29. Veenstra HJ, Vermeeren L, Valdes Olmos RA, Nieweg OE. The additional value of lymphatic mapping with routine SPECT/CT in unselected patients with clinically localized melanoma. Ann Surg Oncol. 2012;19:1018-1023.

30. Vermeeren L, Valdes Olmos RA, Klop MC, et al. SPECT/CT for sentinel node mapping in head and neck melanoma. Head Neck. 2011;33:1-6.

31. Mar MV, Miller SA, Kim EE, Macapinlac HA. Evaluation and localization of lymphatic drainage and sentinel lymph nodes in patients with head and neck melanomas by hybrid SPECT/CT lymphoscintigraphic imaging. J Nucl Med Technol. 2007;35:10-16. 